\title{
Heat shock protein 90 (Hsp90) inhibition targets canonical TGF- $\beta$ signalling to prevent fibrosis
}

\author{
Michal Tomcik, ${ }^{1,2}$ Pawel Zerr, ${ }^{1}$ Jana Pitkowski, ${ }^{1}$ Katrin Palumbo-Zerr, ${ }^{1}$ \\ Jérôme Avouac, ${ }^{1,3}$ Oliver Distler, ${ }^{4}$ Radim Becvar, ${ }^{2}$ Ladislav Senolt, ${ }^{2}$ \\ Georg Schett, ${ }^{1}$ Jörg H Distler ${ }^{1}$
}

\begin{abstract}
Handling editor Tore K Kvien
${ }^{1}$ Department of Internal

Medicine 3 and Institute for

Clinical Immunology, University

of Erlangen-Nuremberg,

Erlangen, Germany

${ }^{2}$ First Faculty of Medicine,

Department of Rheumatology,

Institute of Rheumatology,

Charles University in Prague,

Prague, Czech Republic

${ }^{3}$ Rheumatology A Department and INSERM U1016 Cochin

Hospital, Paris Descartes

University, Paris, France

${ }^{4}$ Center of Experimental

Rheumatology and Zurich

Center of Integrative Human

Physiology, University Hospital

Zurich, Switzerland
\end{abstract}

\section{Correspondence to}

Dr Michal Tomcik, First Faculty of Medicine, Department of

Rheumatology, Institute of

Rheumatology, Charles

University, Na Slupi 4, 12850

Prague 2, Czech Republic;

michaltomcik@yahoo.com

Accepted 14 April 2013

Published Online First

9 May 2013

\begin{tabular}{|l|}
\hline To cite: Tomcik M, Zerr P, \\
Pitkowski J, et al. Ann \\
Rheum Dis 2014;73: \\
1215-1222. \\
\hline
\end{tabular}

\section{ABSTRACT}

Objectives Targeted therapies for systemic sclerosis

(SSc) and other fibrotic diseases are not yet available. We evaluated the efficacy of heat shock protein 90 (Hsp90) inhibition as a novel approach to inhibition of aberrant transforming growth factor (TGF)- $\beta$ signalling and for the treatment of fibrosis in preclinical models of SSc.

Methods Expression of $\mathrm{Hsp} 90$ was quantified by quantitative PCR, western blot and

immunohistochemistry. The effects of Hsp90 inhibition were analysed in cultured fibroblasts, in bleomycininduced dermal fibrosis, in tight-skin (Tsk-1) mice and in mice overexpressing a constitutively active TGF- $\beta$ receptor I (T $\beta \mathrm{RI})$.

Results Expression of Hsp90 $\beta$ was increased in SSc skin and in murine models of SSc in a TGF- $\beta$-dependent manner. Inhibition of Hsp90 by 17-dimethylaminoethylamino-17demethoxy-geldanamycin (17-DMAG) inhibited canonical TGF- $\beta$ signalling and completely prevented the stimulatory effects of TGF- $\beta$ on collagen synthesis and myofibroblast differentiation. Treatment with 17-DMAG decreased the activation of canonical TGF- $\beta$ signalling in murine models of SSc and exerted potent antifibrotic effects in bleomycininduced dermal fibrosis, in Tsk-1 mice and in mice overexpressing a constitutively active T $\beta R I$. Dermal thickness, number of myofibroblasts and hydroxyproline content were all significantly reduced on treatment with 17-DMAG. No toxic effects were observed with 17-DMAG at antifibrotic doses.

Conclusions $\mathrm{Hsp90}$ is upregulated in SSC and is critical for TGF- $\beta$ signalling. Pharmacological inhibition of Hsp90 effectively blocks the profibrotic effects of TGF- $\beta$ in cultured fibroblasts and in different preclinical models of SSc. These results have translational implications, as several Hsp90 inhibitors are in clinical trials for other indications.

\section{INTRODUCTION}

The major hallmark of systemic sclerosis (SSc) is an excessive accumulation of extracellular matrix in affected organs. ${ }^{1}$ The accumulation of extracellular matrix is caused by an increased release of collagen and other components of the extracellular matrix by pathologically activated fibroblasts. ${ }^{2}$ Several lines of evidence suggest a central role of transforming growth factor $\beta$ (TGF- $\beta$ ) in fibroblast activation and tissue fibrosis in SSc: TGF- $\beta$ signalling is activated in SSc with nuclear accumulation of the downstream mediator $S m a d 2 / 3$ and increased transcription of TGF- $\beta$ target genes. ${ }^{3}$ TGF- $\beta$ potently activates fibroblasts and induces an expression profile in resting normal fibroblasts that is reminiscent of SSc fibroblasts. ${ }^{4}$ Moreover, activation of TGF- $\beta$ signallingfor example, by fibroblast-specific overexpression of constitutively active TGF- $\beta$ receptor type I (T $\beta R I)-$ is sufficient to induce fibrosis in mice. ${ }^{5}$ However, the knowledge of the crucial role of TGF- $\beta$ in the pathogenesis of SSc has not yet been successfully translated into molecular therapies, and effective targeted treatments for fibrosis in SSc are still not available for clinical use. ${ }^{6}$

Heat shock proteins (Hsps) are a family of molecular chaperones. They were discovered by virtue of their induction in response to high temperature, but they are also upregulated by other forms of cellular stress. ${ }^{7}$ Functionally, most Hsps selectively recognise and bind non-native proteins under physiological and stress conditions, thereby Hsps prevent irreversible aggregation of proteins. ${ }^{8}$ However, Hsps have additional functions beyond prevention of protein aggregation. They not only modulate antigen processing and presentation to regulate immune responses, ${ }^{9-11}$ but they also interact with a broad variety of different kinases and transcription factors to regulate the cell cycle, apoptosis and cytoskeletal rearrangement. ${ }^{12}$

In this study, we evaluated Hsp90 as a potential target for the treatment of fibrosis in SSc. Hsp90 was selected because of its crucial role in folding and conformational stabilisation of TGF- $\beta$ receptors (T $\beta$ RI and T $\beta$ RII) ${ }^{13}$ and also of Src kinases, which are intracellular mediators of the profibrotic effects of TGF- $\beta .^{14-16}$ Inhibition of Hsp90 accelerates ubiquitination and increases proteasomal degradation of T $\beta R$ and Src. ${ }^{13}$ Thus, inhibition of Hsp90 may be a novel approach to targeting TGF- $\beta$ signalling. Of note, selective Hsp90 inhibitors from more than 10 different companies are currently being evaluated in clinical trials for their efficacy and tolerability in different oncological indications. Results from already completed trials suggest that pharmacological inhibition of Hsp90 is effective and not limited by toxicity. ${ }^{17} 18$ Thus, inhibition of Hsp90 has high translational potential, and various Hsp90 inhibitors would be available for clinical trials in SSc.

\section{MATERIALS AND METHODS}

Patients and fibroblast cultures

Fibroblast cultures were obtained from lesional skin biopsy samples from 15 patients with SSc and 12 healthy volunteers matched for age and sex. All 
patients fulfilled the criteria for SSc as suggested by LeRoy et al. ${ }^{19}$ All had diffuse cutaneous SSc. Additional information is provided in table 1 . The study was approved by the ethics committee of the University of Erlangen-Nuremberg.

Fibroblast cultures from patients and healthy volunteers were cultured as described. ${ }^{20}$ In selected experiments, fibroblasts were stimulated with TGF- $\beta$ at a concentration of $10 \mathrm{ng} / \mathrm{ml}$ (R\&D Systems, Wiesbaden-Nordenstadt, Germany). ${ }^{21}$

\section{Quantitative real-time PCR}

Gene expression was quantified by SYBR Green real-time PCR using the MxPro 3005P QPCR System (Agilent Technologies, Santa Clara, California, USA). ${ }^{21}{ }^{22}$ The following primers were used: human Hsp90 $\beta$, 5'-ACCGCCCTGCTATCTTCTGG CTT- $3^{\prime}$ and 5'-GCATCCTCATCGCCCTCGAGAG-3'; human RPL27, 5'-ATCGCCAAGAGATCAAAGATAA-3' and $5^{\prime}$-TCT GAAGACATCCTTATTGACG-3'. Samples without enzyme in the reverse transcription reaction were used as controls. Human RPL27 was used to normalise for the amounts of cDNA within each sample.

\section{Sodium dodecyl sulphate/polyacrylamide gel electrophoresis and western blotting}

Proteins were separated by sodium dodecyl sulphate/polyacrylamide gel electrophoresis as described. ${ }^{23} 24$ Primary antibodies against Hsp90 (sc-13119; Santa Cruz Biotechnology, Heidelberg, Germany) and $\beta$-actin (Sigma-Aldrich, Hamburg, Germany) and secondary horseradish peroxidase-conjugated goat anti-mouse $\operatorname{IgG}$ or rabbit anti-human $\operatorname{IgG}$ were used.

\section{Immunofluorescence staining}

HSP-90 $\beta$ and phosphorylated (p)Smad2/3 in cultured fibroblasts were detected by incubation with monoclonal mouse antihuman Hsp-90 $\beta$ antibodies (Assaydesigns, Ann Arbor, Michigan, USA) or polyclonal goat anti-human pSmad2/3 antibodies (Santa Cruz Biotechnology). Polyclonal goat anti-mouse Alexa Fluor 488 antibodies (Invitrogen) were used as secondary antibodies. Stress fibres were visualised with rhodamineconjugated phalloidin (Sigma-Aldrich), and the cell nuclei were

Table 1 Clinical characteristics of patients with systemic sclerosis (SSc) at date of biopsy

\begin{tabular}{ll}
\hline Characteristic & Value \\
\hline Gender (F/M) & $11 / 4$ \\
Age (years), median (range) & $52(35-78)$ \\
Disease duration (years), median (range) & $5(0.5-11)$ \\
ANA positive & $15 / 15$ \\
Anti-topoisomerase positive & $6 / 15$ \\
Pulmonary fibrosis & $5 / 15$ \\
Myocardial involvement & $2 / 15$ \\
Pulmonary arterial hypertension & $3 / 15$ \\
Digital ulcers & $8 / 15$ \\
Active disease & $7 / 15$ \\
Medication & No DMARDs, \\
& corticosteroids or NSAIDs
\end{tabular}

All patients had diffuse cutaneous SSc. The disease subset was determined according to the criteria proposed by LeRoy et $a l .^{19}$ Disease duration was measured from the onset of the first non-Raynaud symptoms attributable to SSc. Pulmonary arterial hypertension was diagnosed by right heart catheterisation. Disease activity was determined using the EULAR Systemic Sclerosis Activity Score. Patients with scores of $\geq 3$ were classified as having active disease.

DMARD, disease-modifying antirheumatic drug; F, female; M, male; NSAID,

non-steroidal anti-inflammatory drug. stained using 4',6-diamidino-2-phenylindole (1:800; Santa Cruz Biotechnology). Images were captured at 200-fold magnification using a Nikon Eclipse 80i microscope (Nikon, Badhoevedorp, The Netherlands).

\section{Immunohistochemistry staining}

Formalin-fixed, paraffin-embedded skin sections were stained with mouse anti-human Hsp90 $\beta$ or mouse anti-human $\alpha$-smooth muscle actin $(1: 500$; clone $1 \mathrm{~A} 4$; Sigma-Aldrich) antibodies. Peroxidase-labelled species-specific immunoglobulins (Dako, Glostrup, Denmark) were used as secondary antibodies, and isotype-matched antibodies were used as controls.

\section{Quantification of soluble collagen in cell culture supernatants}

Soluble collagen in cell culture supernatants was quantified using the SirCol collagen assay (Biocolor, Belfast, Northern Ireland) as described. ${ }^{25}$

\section{T及RI-induced dermal fibrosis}

Injection of replication-deficient adeno-associated virus (AAVs) encoding constitutively active TRRI (AdTBR) into the skin of mice induces dermal fibrosis. ${ }^{26} 27$ AdTBR or AdLacZ $\left(6.7 \times 10^{7}\right.$ infectious units (ifu)/ml) was injected intracutaneously into defined areas of the upper back of 4-week-old FVB mice at day 1 and day 28. Treatment with 17-dimethylaminoethylamino-17demethoxy-geldanamycin (17-DMAG; $5 \mathrm{mg} / \mathrm{ml}$ in phosphatebuffered saline; InvivoGen, San Diego, California, USA) was started at day 1 and continued every third day for 8 weeks. After 8 weeks, mice were killed, and dermal thickness, number of myofibroblasts and hydroxyproline content were analysed as described. $^{24} 28-31$

\section{Bleomycin-induced dermal fibrosis}

Skin fibrosis was induced in 6-week-old DBA/2J mice by local injection of bleomycin every other day for 4 weeks. ${ }^{30} 32$ One group of mice challenged with bleomycin was intraperitoneally injected with $100 \mu \mathrm{l}$ 17-DMAG every third day for 4 weeks. The control NaCl- and bleomycin-treated groups were injected intraperitoneally with $100 \mu \mathrm{l}$ phosphate-buffered saline every third day for 4 weeks.

\section{Tsk-1 mouse model of dermal fibrosis}

The tight-skin (Tsk-1) phenotype is induced by a dominant mutation in the fibrillin-1 gene, which results in increased activation of latent TGF- $\beta .^{33}$ TSK- 1 mice were interbred with $\mathrm{pa} / \mathrm{pa}$ mice, in which a recessive mutation (pa) induces a light grey color of the fur and pink eyes. Because the fibrillin 1 gene is genetically linked to the pa gene, mice can be prescreened for the tsk1 mutation based on the color of their fur and eyes. All mice with black fur and eyes carry the dominant $t s k 1$ mutation and are heterozygous for the pale mutation. In contrast, mice with light grey fur do not carry the $t s k 1$ mutation but are homozygous for the mutated pale gene. Apart from the change in skin color, the pale mutation itself does not alter skin physiology or fibrogenesis. ${ }^{34}$ Treatment with 17-DMAG was started at the age of 5 weeks, and mice were killed after 5 weeks of treatment.

\section{Statistical analysis}

All data are presented as median with IQR, and differences between the groups were tested for their statistical significance by non-parametric Mann-Whitney U test. A p value of less than 0.05 was considered significant. 


\section{RESULTS}

\section{Hsp90ß is upregulated in SSc fibroblasts}

Hsp90 $\beta$ was induced in the skin of patients with SSc compared with healthy individuals (figure 1A). In healthy volunteers, Hsp90 $\beta$ was either undetectable (3/8 individuals) or expressed at low levels with faint staining of the dermis (5/8 individuals). In contrast, prominent staining for $\mathrm{Hsp} 90 \beta$ was detected in fibroblasts of the upper dermal layer of all patients with SSc (figure 1A). Keratinocytes and endothelial cells also stained positive for Hsp90 3 in three out of eight and six out of eight patients, respectively. Of particular interest, expression of $\mathrm{Hsp} 90 \mathrm{\beta}$ in fibroblasts colocalised with expression of $\alpha$-smooth muscle actin (data not shown). Indeed, all myofibroblasts stained intensely for Hsp90 $\beta$ in SSc skin. Although not statistically significant, the mRNA levels of Hsp90 $\beta$ tended to be higher in patients with high disease activity as indicated by a European League Against Rheumatism (EULAR) Systemic Sclerosis Activity Score $\geq 3$ (increase of $22 \%, p=0.10$ ). No differences were observed between patients with and without antibodies to topoisomerase. However, further studies on larger patient collectives are needed to determine differences in Hsp90 expression between different subgroups of patients with diffuse cutaneous SSc.
Consistent with the findings in human SSc, Hsp90 $\beta$ was also induced in the mouse model of bleomycin-induced fibrosis and in Tsk-1 mice (figure 1B,C).

We next analysed whether the upregulation of Hsp90 $\beta$ in SSc is due to increased TGF- $\beta$. The basal mRNA and protein levels of $\mathrm{Hsp} 90$ in the absence of exogenous stimulation were increased in cultured SSc fibroblasts compared with fibroblasts from healthy individuals. Consistent with persistent activation of TGF- $\beta$ signalling in cultured SSc fibroblasts, the stimulatory effects of TGF- $\beta$ on Hsp90 mRNA and protein were more pronounced in fibroblasts from healthy individuals than in SSc fibroblasts (figure 1D,E). The stimulatory effects of TGF- $\beta$ signalling on Hsp90 $\beta$ were confirmed in vivo. Activation of TGF- $\beta$ signalling by overexpression of a constitutively active T $\beta R I$ upregulated the expression of $\mathrm{Hsp} 90 \beta$ in murine skin (figure $1 \mathrm{~F}$ ).

\section{Inhibition of Hsp90 abrogates the stimulatory effects of TGF- $\beta$ on fibroblasts}

We next evaluated whether Hsp90 regulates the profibrotic effects of TGF- $\beta$. We first analysed whether the selective Hsp90 inhibitor, 17-DMAG, prevents the nuclear accumulation of $\mathrm{pSmad} 2 / 3$ on stimulation with TGF- $\beta$. Indeed, treatment with
A
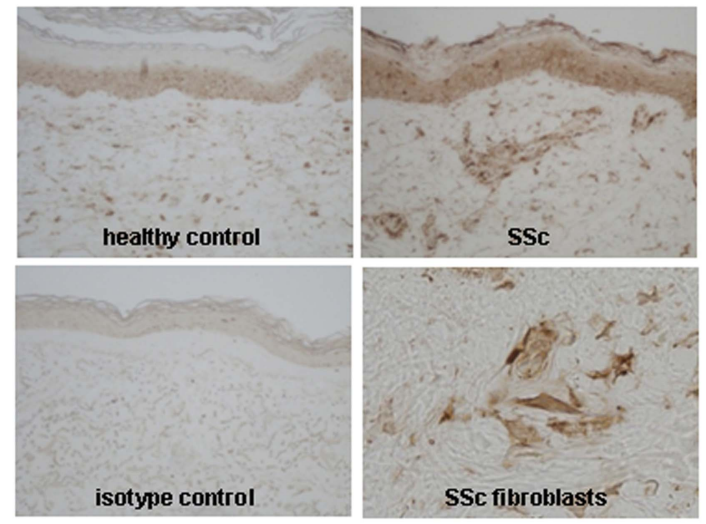

D

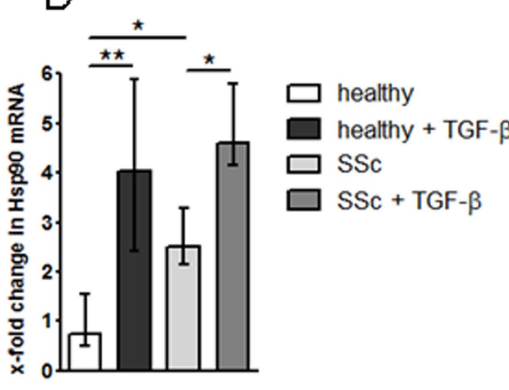

E

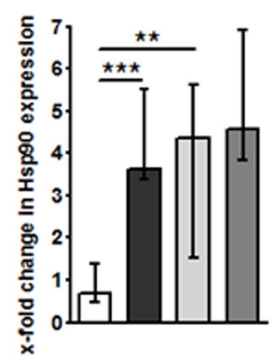

B

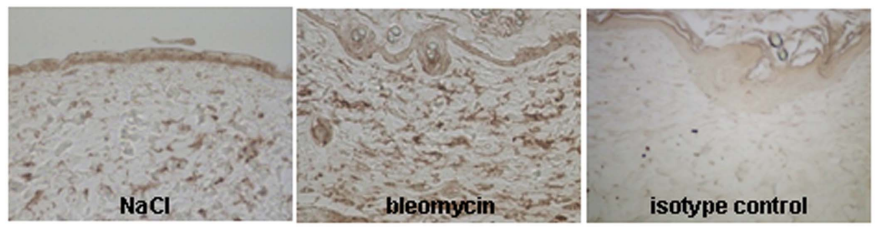

$\mathrm{C}$

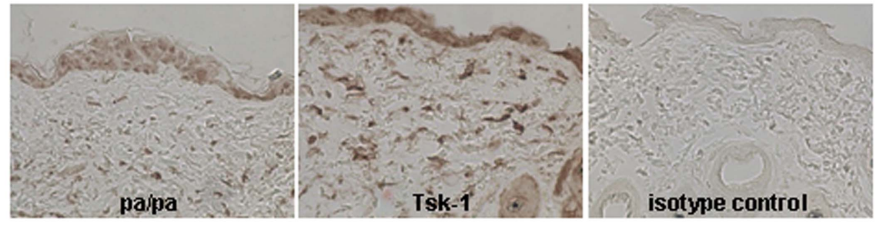

$\mathrm{F}$

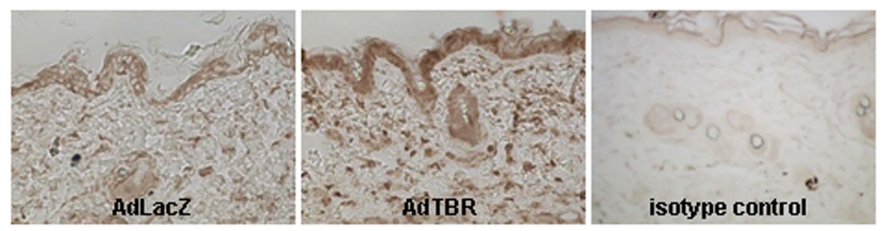

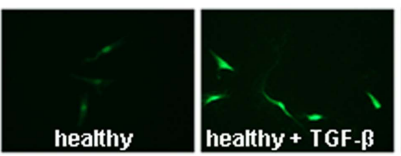

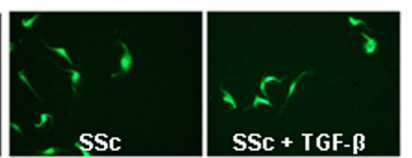

Figure 1 Heat shock protein 90 (Hsp90) is increased in patients with systemic sclerosis (SSc) and in experimental fibrosis in a transforming growth factor $\beta$ (TGF- $\beta$ )-dependent manner. Increased expression of Hsp90 $\beta$ was detected by immunohistochemistry in the upper layer of the dermis of patients with SSC as shown in representative images at 200-fold magnification (A). The levels of Hsp90 $\beta$ are also upregulated in bleomycin-induced skin fibrosis (B) and in Tsk-1 mice (C). The stimulatory effects of TGF- $\beta$ on Hsp90 mRNA (D) and protein levels (E) are more pronounced in fibroblasts from healthy individuals than in fibroblasts from patients with SSc. The expression of Hsp90 $\beta$ is also increased in the skin of mice infected with adenovirus overexpressing constitutively active TGF- $\beta$ receptor I (AdTBR) compared with mice infected with LacZ virus as shown in the representative images at 400-fold magnification (F). $p$ Values are expressed as follows: $0.05>p>0.01$ as *; $0.01>p>0.001$ as **; $p<0.001$ as ***. All data are presented as median with interquartile range (IQR). This figure is only reproduced in colour in the online version. 
A

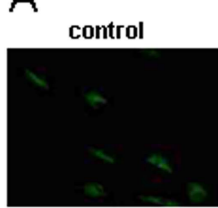

TGF- $\beta$ + 17-DMAG
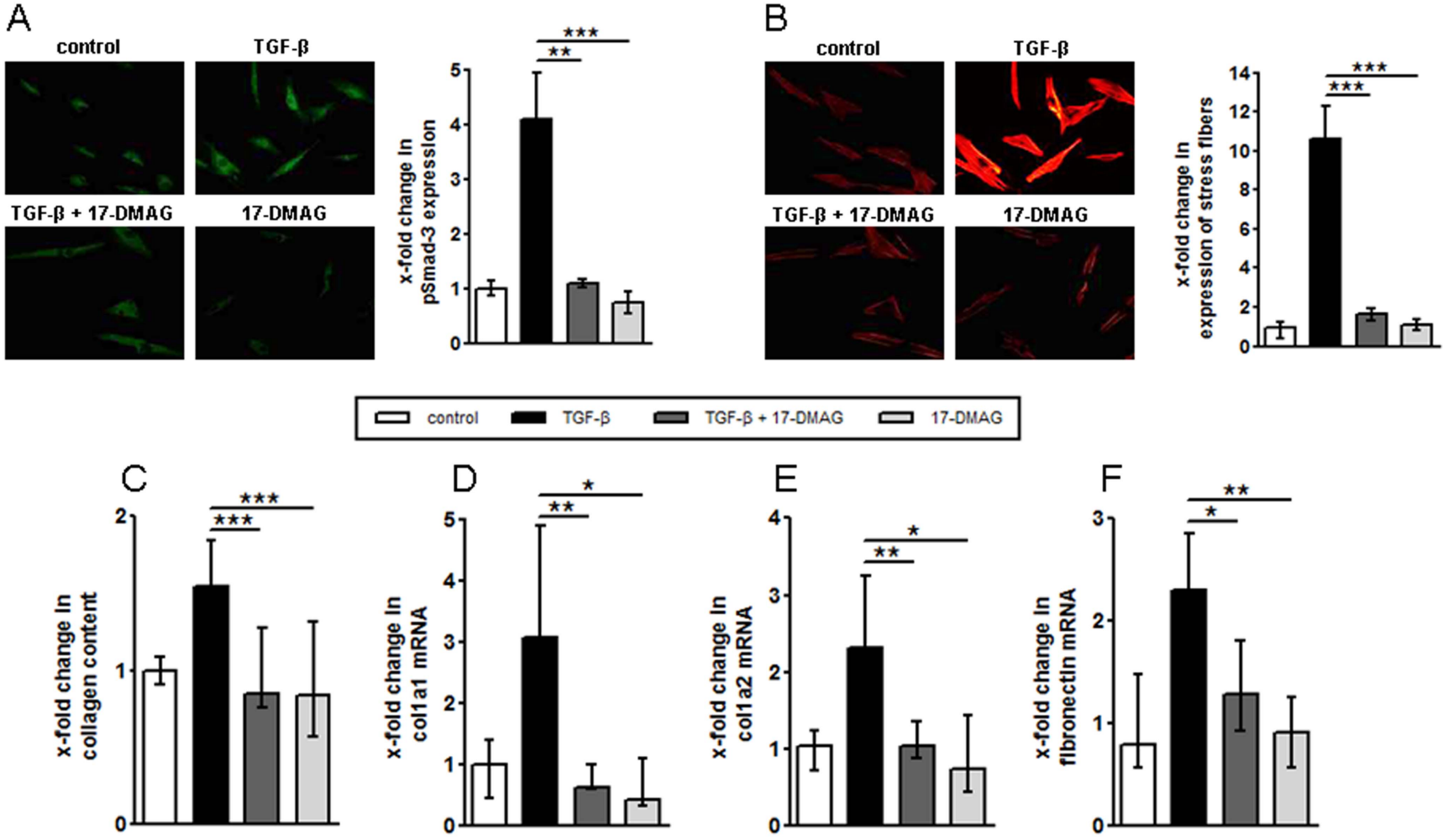

Figure 2 Inhibition of heat shock protein 90 (Hsp90) abrogates stimulatory effects of transforming growth factor $\beta$ (TGF- $\beta$ ) on collagen release in fibroblasts. 17-Dimethylaminoethylamino-17-demethoxy-geldanamycin (17-DMAG) inhibited canonical Smad signalling and prevented accumulation of phosphorylated ( $p$ )Smad2/3 (A). 17-DMAG reduced the stimulatory effects of TGF- $\beta$ on stress fibre formation (B), on the release of collagen protein (C) and on the mRNA levels of col1a1 (D), col1a2 (E) and fibronectin (F). Number of cell lines per experiment: four for $A, B$ and nine for C-F. $p$ values are expressed as follows: $0.05>p>0.01$ as *; $0.01>p>0.001$ as **; $p<0.001$ as ***. All data are presented as median with interquartile range (IQR). This figure is only reproduced in colour in the online version.

17-DMAG abrogated the nuclear accumulation of $\mathrm{pSmad} 2 / 3$ in TGF- $\beta$-stimulated fibroblasts $(p<0.01)$ (figure $2 A)$. Formation of stress fibres is a hallmark of myofibroblasts and is induced by TGF- $\beta$. Preincubation with 17-DMAG prevented the TGF- $\beta$-induced formation of stress fibres $(p<0.001)$ (figure $2 B$ ). Moreover, 17-DMAG effectively inhibited the stimulatory effects of TGF- $\beta$ on the collagen synthesis in dermal fibroblasts. The TGF- $\beta$-induced increase in secreted collagen protein and mRNA levels of col1a1 and col1a2 was completely abrogated $(\mathrm{p}<0.001, \mathrm{p}<0.01$ and $\mathrm{p}<0.01$, respectively) (figure $2 \mathrm{C}-\mathrm{E})$. In addition, the mRNA levels of fibronectin were decreased by $72 \%$ in 17-DMAG-treated cells compared with sham-treated fibroblasts stimulated with TGF- $\beta(p<0.05)$ (figure $2 F$ ).

\section{Inhibition of Hsp90 prevents fibrosis induced by overexpression of TBRI}

After demonstrating that Hsp90 inhibition reduces the profibrotic effects of TGF- $\beta$ in vitro, we next aimed to confirm these findings in vivo. We thus evaluated the effects of 17-DMAG in the mouse model of T $\beta R I$-driven fibrosis, in which fibrosis is induced by selective activation of TGF- $\beta$ signalling. ${ }^{33}$ Inhibition of Hsp90 exerted potent antifibrotic effects in AdTBR mice (figure 3A-E). Treatment with 17-DMAG reduced dermal thickening by $81 \%$ compared with sham-treated AdTBR mice $(p<0.001)$ (figure 3A,B). Hydroxyproline content and myofibroblast count were also significantly decreased by $88 \%$ $(\mathrm{p}<0.05)$ and $69 \%(\mathrm{p}<0.01)$, respectively, on treatment with 17-DMAG (figure 3C,D).
The antifibrotic effects of Hsp90 inhibition were accompanied by potent inhibition of canonical TGF- $\beta$. The nuclear accumulation of $\mathrm{pSmad} 2 / 3$ was reduced by $55 \%$ in 17-DMAG-treated AdTBR mice compared with sham-treated AdTBR mice $(p<0.001)$ (figure 3E).

\section{Treatment with 17-DMAG protects from bleomycin-induced fibrosis}

To further evaluate the antifibrotic potential of the Hsp90 inhibitor in vivo, we used the mouse model of bleomycin-induced dermal fibrosis, which serves as a model for early, inflammatory stages of SSc. ${ }^{33}$ Treatment with 17-DMAG reduced dermal thickening by $56 \% \quad(\mathrm{p}<0.001$ compared with sham-treated, bleomycin-challenged mice) (figure 4A,B). Furthermore, the hydroxyproline content in lesional skin was reduced by $81 \%$ $(\mathrm{p}<0.05)$ (figure 4C). The differentiation of resting fibroblasts into myofibroblasts was reduced by $65 \%(p<0.001)$ compared with sham-treated, bleomycin-challenged mice (figure 4D). Hsp90 inhibition effectively reduced canonical TGF- $\beta$ signalling with a $74 \%$ decrease in the number of cells positive for $\mathrm{pSmad} 2 /$ $3(\mathrm{p}<0.001)$ (figure 4E).

\section{Inhibition of Hsp90 ameliorates fibrosis in Tsk-1 mice}

To confirm the antifibrotic effects of Hsp90 inhibition in another, less inflammatory mouse model that resembles later, non-inflammatory stages of fibrosis, ${ }^{33} 34$ Tsk-1 mice were treated with 17-DMAG. Inhibition of Hsp90 reduced hypodermal thickening in Tsk-1 mice by $76 \%$ compared with shamtreated Tsk-1 mice $(\mathrm{p}<0.001)$ (figure 5A,B). Hydroxyproline 

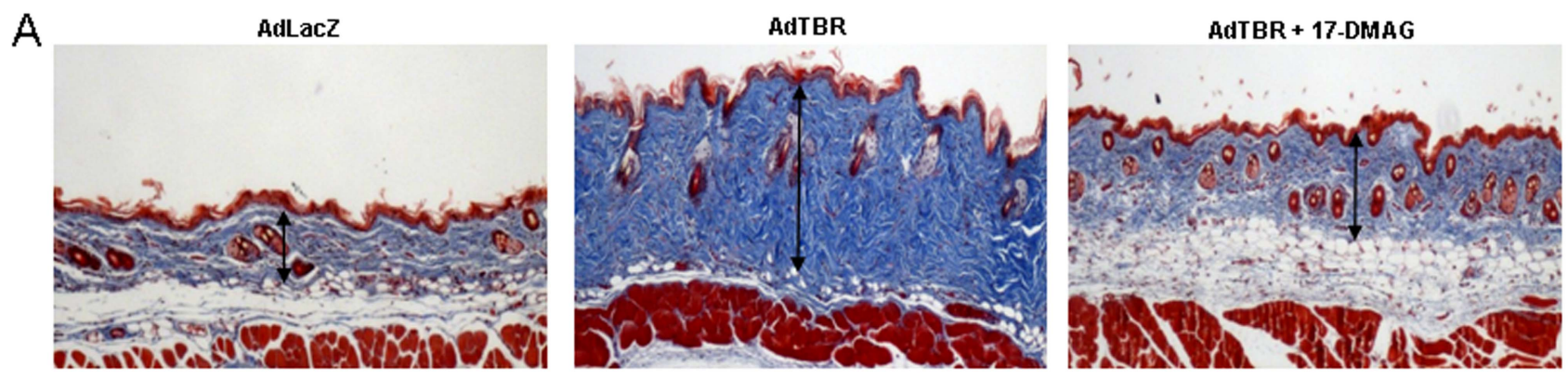

$\sqsubseteq$ AdLacZ $\rightleftharpoons$ AdTBR
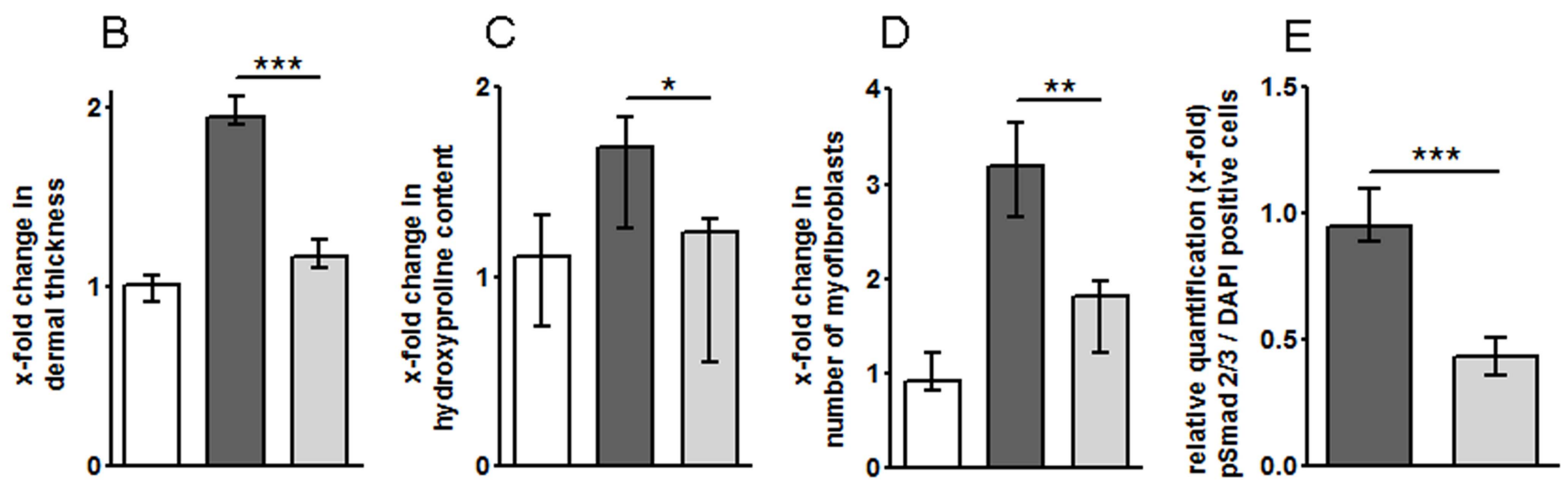

Figure 3 17-Dimethylaminoethylamino-17-demethoxy-geldanamycin (17-DMAG) prevents AdTBR-induced skin fibrosis. Inhibition of heat shock protein 90 (Hsp90) reduced dermal thickening (A, B), hydroxyproline content (C) and numbers of myofibroblasts (D) in AdTBR mice. 17-DMAG reduced transforming growth factor $\beta$ (TGF- $\beta$ ) signalling as shown by reduced nuclear accumulation of phosphorylated ( $p$ )Smad2/3 (E). Representative images of trichrome-stained sections are shown at 100-fold magnification. Seven mice were used for each group (AdLacZ, AdTBR, AdTBR+17-DMAG). $p$ values are expressed as follows: $0.05>p>0.01$ as *; $0.01>p>0.001$ as **; $p<0.001$ as ***. All data are presented as median with interquartile range (IQR). DAPI, 4',6-diamidino-2-phenylindole. This figure is only reproduced in colour in the online version.

content and myofibroblast count were also reduced by $67 \%$ $(\mathrm{p}<0.05)$ and $85 \%(\mathrm{p}<0.001)$, respectively, in Tsk-1 mice treated with 17-DMAG (figure 5C,D). Similar to previous models, 17-DMAG inhibited canonical TGF- $\beta$ signalling and reduced the number of cells with nuclear $\mathrm{pSmad} 2 / 3$ by $49 \%$ $(\mathrm{p}<0.001)$ compared with sham-treated Tsk-1 mice (figure 5E). To exclude the possibility that the antifibrotic effects of 17-DMAG are accompanied by major toxic side effects, mice in all models were closely monitored during the treatment. No clinical signs of toxicity were observed, and the mean body weight, activity of the mice and texture of the fur were not altered in mice treated with antifibrotic doses of 17-DMAG for up to 8 weeks. Moreover, gross macroscopic evaluation of the mice on necropsy revealed no signs of toxicity (data not shown).

\section{DISCUSSION}

We demonstrate in this study that inhibition of Hsp90 abrogates the profibrotic effects of TGF- $\beta$ signalling. Incubation of cultured fibroblasts with 17-DMAG prevented the nuclear accumulation of $\mathrm{pSmad} 2 / 3$, inhibited the differentiation of resting fibroblasts into myofibroblasts and completely abrogated the stimulatory effects of TGF- $\beta$ on the release of extracellular matrix in vitro. Pharmacological inhibition of Hsp90 also effectively attenuated TGF- $\beta$ signalling in vivo and significantly reduced skin fibrosis induced by overexpression of a constitutively active T $\beta$ RI. Hsp90 is required for correct folding and conformational stabilisation of $\mathrm{T} \beta \mathrm{Rs}$ and $\mathrm{Src}$ kinases. ${ }^{13}$ Although we did not directly measure the activity of Hsp90 in fibrotic skin, the potency of the effects of Hsp90 inhibition with complete abrogation of the profibrotic effects of TGF- $\beta$ signalling in vitro and the efficacy of 17-DMAG in short-term experiments suggest that Hsp90 controls TGF- $\beta$ signalling in fibroblasts also via other mechanisms in addition to conformational stabilisation. Indeed, Hsps have been recently suggested to directly affect the activity of kinases. In this context, JNK1/ 2 and JAK2 kinases may be interesting candidates as mediators of the effects of Hsp90 on TGF- $\beta$ signalling, because (i) they have been shown to be regulated by Hsp90 $0^{35} 36$ and (ii) they are established downstream mediators of the profibrotic effects of TGF- $\beta$ in fibroblasts. ${ }^{28} 37$ However, further studies on Hsp90 activity and on its effects on JNK and JAK kinases are needed to elucidate how Hsp90 regulates TGF- $\beta$ signalling in fibroblasts.

Hsp90 is overexpressed in patients with SSc and in murine models of skin fibrosis. The upregulation of Hsp90 in AdTBR mice suggests that increased TGF- $\beta$ signalling may be an important factor for the upregulation of $\mathrm{Hsp} 90$ in fibrosis. TGF- $\beta$ stimulated the transcription and increased the protein levels of Hsp90 $\beta$ in cultured fibroblasts. Increased TGF- $\beta$ signalling also stimulated the expression of $\mathrm{Hsp} 90 \beta$ in fibroblasts in murine skin. As Hsp90 is required for effective TGF- $\beta$ signalling, Hsp90 serves as an intracellular amplifier of the profibrotic effects of TGF- $\beta$ in SSc. TGF- $\beta$ increases the expression of Hsp90, which in turn renders fibroblasts more susceptible to TGF- $\beta$ signalling by stabilising TGF- $\beta$ cell surface receptors and intracellular downstream mediators such as Src, JNK and JAK kinases. The disruption of this endogenous amplification loop and the interference with TGF- $\beta$ signalling 

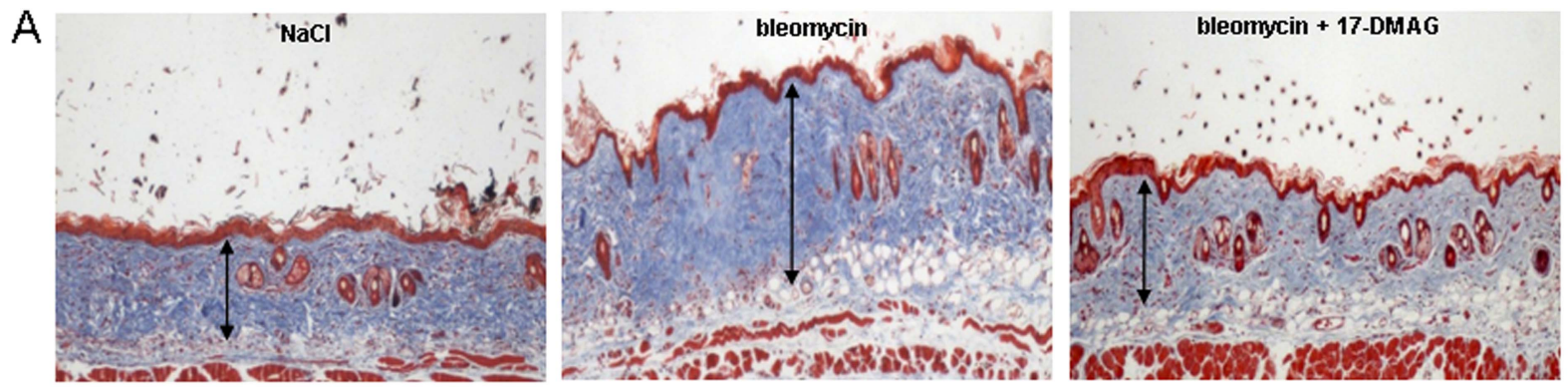

$\varpi \mathrm{NaCl} \rightleftharpoons$ bleomycin bleomycin + 17-DMAG

B

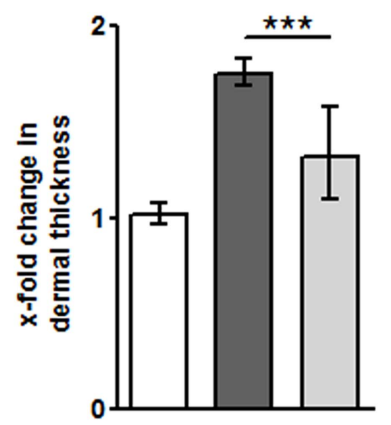

C

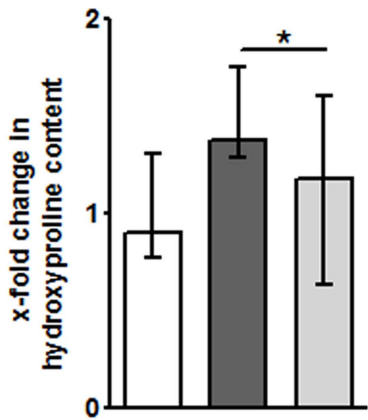

D

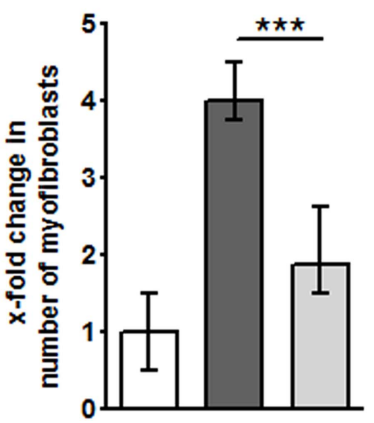

E

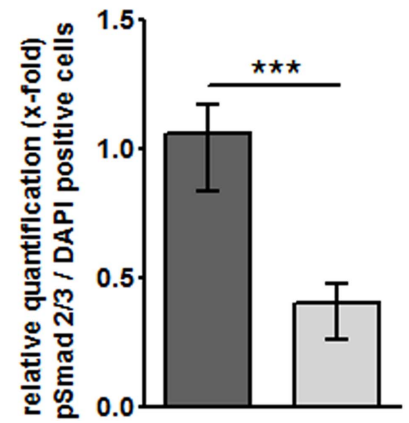

Figure 4 Inhibition of heat shock protein 90 (Hsp90) reduced bleomycin-induced fibrosis. Treatment with 17-dimethylaminoethylamino-17demethoxy-geldanamycin (17-DMAG) prevents dermal thickening $(A, B)$, accumulation of hydroxyproline $(C)$ and myofibroblast differentiation (D) and reduces the nuclear accumulation of phosphorylated $(\mathrm{p}) \operatorname{Smad} 2 / 3(\mathrm{E})$ in bleomycin-challenged mice. Representative images of trichrome-stained sections are shown at 100-fold magnification. Number of mice used: $\mathrm{NaCl}_{1}{ }^{12}$; bleomycin ${ }^{9} ;$; bleomycin+17-DMAG, ${ }^{9}$. p values are expressed as follows: $0.05>\mathrm{p}>0.01$ as *; $\mathrm{p}<0.001$ as ${ }^{* * *}$. All data are presented as median with interquartile range (IQR). DAPI, 4',6-diamidino-2-phenylindole. This figure is only reproduced in colour in the online version.
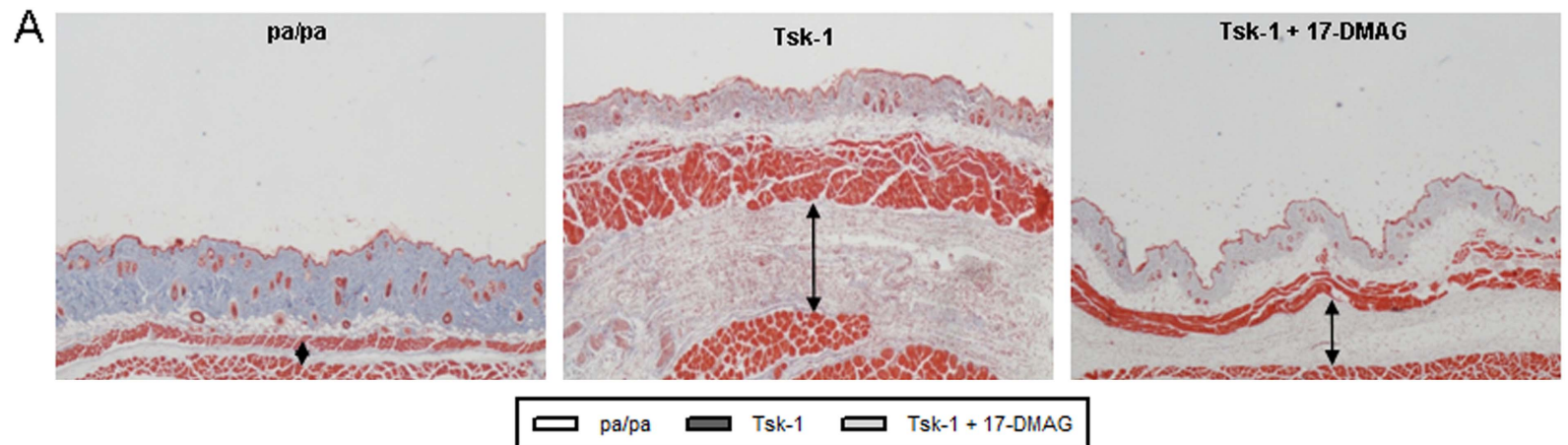

B

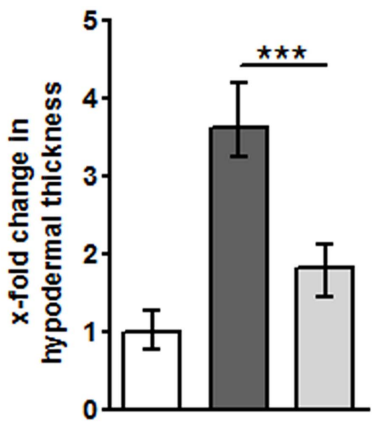

C

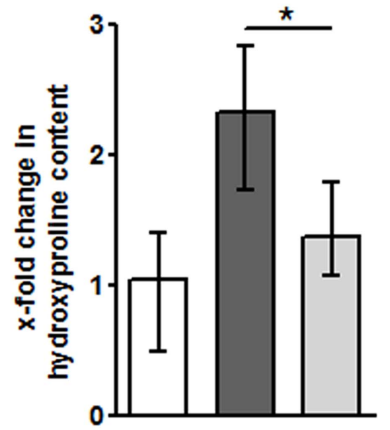

D

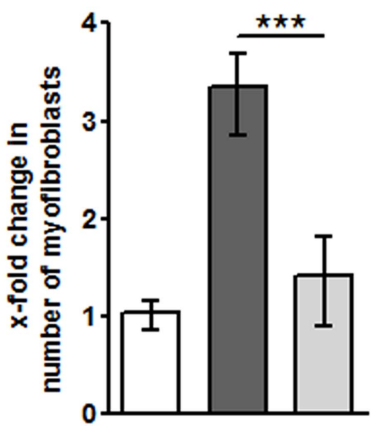

E

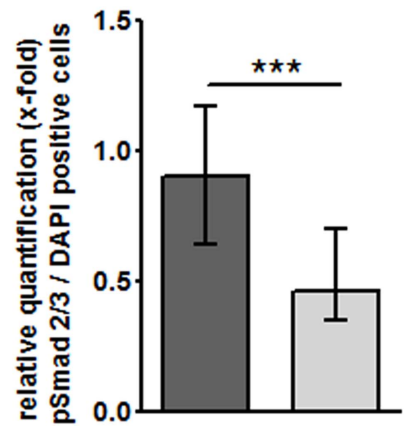

Figure 5 17-Dimethylaminoethylamino-17-demethoxy-geldanamycin (17-DMAG) reduces fibrosis in the Tsk-1 mouse model. Pharmacological inhibition of heat shock protein 90 (Hsp90) by 17-DMAG reduces hypodermal thickening $(A, B)$, decreases the hydroxyproline content (C), inhibits myofibroblast differentiation (D) and prevents nuclear accumulation of phosphorylated (p)Smad2/3 (E) in Tsk-1 mice. Representative images of trichrome-stained sections are shown at 40-fold magnification. Number of mice used: pa/pa, 7; Tsk-1, 6; Tsk-1+17-DMAG, 6 . p values are expressed as follows: $0.05>p>0.01$ as ${ }^{*} ; p<0.001$ as ${ }^{* * *}$. All data are presented as median with interquartile range (IQR). DAPI, 4',6-diamidino-2phenylindole. 
on multiple levels may explain the potent antifibrotic effects of Hsp90 inhibitors.

We demonstrated that pharmacological inhibition of Hsp90 strongly inhibits TGF- $\beta$ signalling as a core pathway of fibrosis in vitro and in vivo. The inhibitory effects on TGF- $\beta$ signalling translate into potent antifibrotic effects. Treatment with the Hsp90 inhibitor, 17-DMAG, significantly reduced histological features of fibrosis, decreased collagen content and prevented the differentiation of resting fibroblasts into myofibroblasts in three different mouse models in pharmacologically relevant doses. The mouse models used herein mimic different stages and aspects of SSc. The mouse model of bleomycin-induced dermal fibrosis resembles early, inflammatory stages of SSc, in which fibroblasts are predominantly activated by profibrotic mediators released from infiltrating leucocytes. ${ }^{33} 38$ In contrast, Tsk-1 mice represent later stages of SSc, in which inflammatory infiltrates have already resolved and fibroblasts have become endogenously activated. ${ }^{33} 39$ In addition, the Tsk-1 model also highlights the key role of B cells in SSc. ${ }^{40}$ The AdTBR model is independent of inflammation, and fibrosis is exclusively driven by enhanced TGF- $\beta$ signalling in fibroblasts. ${ }^{5} 33$ The potent antifibrotic effects of 17-DMAG in all of these models indicate that Hsp90 inhibition may be effective in different fibrotic conditions and at different stages of SSc. Of note, antifibrotic doses of 17-DMAG were well tolerated and toxic effects were neither observed by clinical monitoring nor on necroscopic evaluation. The tolerability of Hsp90 inhibitors has been further evaluated by numerous clinical trials with different Hsp90 inhibitors for various oncological indications during the last two decades. The first-in-class Hsp90 inhibitor, 17-AAG (17-N-allylamino-17demethoxygeldanamycin), entered the first clinical trials in 1999. The promising results with this inhibitor stimulated the development of second-generation Hsp90 inhibitors such as alvespimycin (KOS-1022, 17-DMAG) with improved pharmacokinetics. Hsp90 inhibitors were initially evaluated for the treatment of several solid tumours, such as breast, prostate and lung cancers, but have more recently also been tested in haematological malignancies, such as chronic myeloid leukaemia and multiple myeloma. Moreover, recent trials have also evaluated the effects of combination therapies of Hsp90 inhibitors with either other targeted therapies or conventional chemotherapy or radiotherapy. ${ }^{41}$ Currently, 13 different Hsp90 inhibitors from several pharmaceutical companies in various oncological indications are ongoing (http://www.clinicaltrials.gov). ${ }^{42} 43$ Together, these data indicate that an application in patients with fibrotic diseases might not be limited by toxicity.

In summary, we demonstrate that Hsp90 amplifies the profibrotic effects of TGF- $\beta$ and that pharmacological inhibition of Hsp90 potently inhibits fibrosis in preclinical models of different stages of SSc in pharmacologically relevant doses without obvious toxicity. These findings might have direct translational implications. Hsp90 is currently considered a prime candidate for the treatment of various cancers, and its good drugability and encouraging results from clinical trials have stimulated multiple companies to develop Hsp90 inhibitors. Selective and potent inhibitors from about a dozen companies are currently in clinical trial and would be available for clinical trials in SSc and other fibrosing diseases.

Acknowledgements We thank Madeleine Demleitner, Maria Halter and Verena Wäsch for excellent technical assistance.

Contributors Design of the study: MT, JHD. Acquisition of data: MT, PZ, JP, KPZ, JA. Interpretation of data: MT, PZ, JP, KPZ, JA, OD, RB, LS, GS, JHD. Manuscript preparation: MT, PZ, OD, RB, LS, GS, JHD.
Funding Grants DI 1537/4-1, DI 1537/5-1, AK 144/1-1 and SCHE 1583/7-1 from the Deutsche Forschungsgesellschaft, grant A40 from the IZKF in Erlangen, the ELAN-Program of the University of Erlangen-Nuremberg and the Career Support Award of Medicine from the Ernst Jung Foundation, Ministry of Health, Czech Republic (Research Project No 00023728) and SVV: 262512.

Competing interests None.

Ethics approval Ethics committee of the University of Erlangen-Nuremberg.

Provenance and peer review Not commissioned; externally peer reviewed.

\section{REFERENCES}

1 Gabrielli A, Avvedimento EV, Krieg T. Scleroderma. N Engl J Med 2009:360:1989-2003.

2 Varga J, Abraham D. Systemic sclerosis: a prototypic multisystem fibrotic disorder. J Clin Invest 2007;117:557-67.

3 Mori Y, Chen SJ, Varga J. Expression and regulation of intracellular SMAD signaling in scleroderma skin fibroblasts. Arthritis Rheum 2003;48:1964-78.

4 Whitfield ML, Finlay DR, Murray Jl, et al. Systemic and cell type-specific gene expression patterns in scleroderma skin. Proc Natl Acad Sci USA 2003; 100:12319-24.

5 Sonnylal $S$, Denton $C P$, Zheng $B$, et al. Postnatal induction of transforming growth factor beta signaling in fibroblasts of mice recapitulates clinical, histologic, and biochemical features of scleroderma. Arthritis Rheum 2007;56:334-44.

6 Charles C, Clements P, Furst DE. Systemic sclerosis: hypothesis-driven treatment strategies. Lancet 2006;367:1683-91.

7 Hartl FU. Molecular chaperones in cellular protein folding. Nature 1996:381:571-9.

8 Buchner J. Supervising the fold: functional principles of molecular chaperones. FASEB J 1996;10:10-19.

9 Zügel U, Kaufmann SH. Role of heat shock proteins in protection from and pathogenesis of infectious diseases. Clin Microbiol Rev 1999;12:19-39.

10 De Nagel DC, Pierce SK. Heat shock proteins implicated in antigen processing and presentation. Semin Immunol 1991;3:65-71.

11 Srivastava P. Roles of heat-shock proteins in innate and adaptive immunity. Nat Rev Immunol 2002;2:185-94.

12 Nardai G, Végh EM, Prohászka Z, et al. Chaperone-related immune dysfunction: an emergent property of distorted chaperone networks. Trends Immunol 2006:27:74-9.

13 Wrighton $\mathrm{KH}$, Lin X, Feng XH. Critical regulation of TGFbeta signaling by Hsp90. Proc Natl Acad Sci USA 2008;105:9244-9.

14 Koga F, Xu W, Karpova TS, et al. Hsp90 inhibition transiently activates Src kinase and promotes Src-dependent Akt and Erk activation. Proc Natl Acad Sci USA 2006;103:11318-22.

15 Skhirtladze C, Distler O, Dees C, et al. Src kinases in systemic sclerosis: central roles in fibroblast activation and in skin fibrosis. Arthritis Rheum 2008;58: 1475-84

16 Beyer C, Distler JH. Tyrosine kinase signaling in fibrotic disorders: translation of basic research to human disease. Biochim Biophys Acta 2013;1832:897-904.

17 Soga S, Akinaga S, Shiotsu Y. Hsp90 inhibitors as anti-cancer agents, from basic discoveries to clinical development. Curr Pharm Des 2013;19:366-76.

18 Nahleh Z, Tfayli A, Najm A, et al. Heat shock proteins in cancer: targeting the 'chaperones'. Future Med Chem 2012;4:927-35.

19 LeRoy EC, Black C, Fleischmajer R, et al. Scleroderma (systemic sclerosis): classification, subsets and pathogenesis. J Rheumatol 1988;15:202-5.

20 Dees $C$, Akhmetshina A, Zerr $P$, et al. Platelet-derived serotonin links vascular disease and tissue fibrosis. J Exp Med 2011;208:961-72.

21 Distler JH, Jüngel A, Huber LC, et al. Imatinib mesylate reduces production of extracellular matrix and prevents development of experimental dermal fibrosis. Arthritis Rheum 2007;56:311-22.

22 Lin NY, Beyer C, Gießl A, et al. Autophagy regulates TNF $\alpha$-mediated joint destruction in experimental arthritis. Ann Rheum Dis 2013;72:761-8.

23 Schaffner W, Weissmann C. A rapid, sensitive, and specific method for the determination of protein in dilute solution. Anal Biochem 1973;56:502-14.

24 Krämer M, Dees C, Huang J, et al. Inhibition of H3K27 histone trimethylation activates fibroblasts and induces fibrosis. Ann Rheum Dis 2013;72:614-20.

25 Palumbo K, Zerr P, Tomcik M, et al. The transcription factor JunD mediates transforming growth factor \{beta\}-induced fibroblast activation and fibrosis in systemic sclerosis. Ann Rheum Dis 2011;70:1320-6.

26 Akhmetshina A, Palumbo K, Dees C, et al. Activation of canonical Wnt signalling is required for TGF- $\beta$-mediated fibrosis. Nat Commun 2012;3:735.

27 Avouac J, Palumbo K, Tomcik M, et al. Inhibition of activator protein 1 signaling abrogates transforming growth factor $\beta$-mediated activation of fibroblasts and prevents experimental fibrosis. Arthritis Rheum 2012;64:1642-52.

28 Dees C, Zerr P, Tomcik M, et al. Inhibition of Notch signaling prevents experimental fibrosis and induces regression of established fibrosis. Arthritis Rheum 2011:63:1396-404.

29 Beyer $C$, Schramm A, Akhmetshina A, et al. $\beta$-catenin is a central mediator of pro-fibrotic Wnt signaling in systemic sclerosis. Ann Rheum Dis 2012;71:761-7. 
30 Beyer $\mathrm{C}$, Reich $\mathrm{N}$, Schindler SC, et al. Stimulation of soluble guanylate cyclase reduces experimental dermal fibrosis. Ann Rheum Dis 2012;71:1019-26.

31 Zerr P, Palumbo-Zerr K, Distler A, et al. Inhibition of hedgehog signaling for the treatment of murine sclerodermatous chronic graft-versus-host disease. Blood 2012;120:2909-17.

32 Akhmetshina A, Dees C, Pileckyte M, et al. Dual inhibition of c-abl and PDGF receptor signaling by dasatinib and nilotinib for the treatment of dermal fibrosis. FASEB J 2008;22:2214-22.

33 Beyer C, Schett G, Distler 0 , et al. Animal models of systemic sclerosis: prospects and limitations. Arthritis Rheum 2010;62:2831-44.

34 Akhmetshina A, Venalis P, Dees C, et al. Treatment with imatinib prevents fibrosis in different preclinical models of systemic sclerosis and induces regression of established fibrosis. Arthritis Rheum 2009;60:219-24.

35 Haupt A, Joberty G, Bantscheff $M$, et al. Hsp90 inhibition differentially destabilises MAP kinase and TGF-beta signalling components in cancer cells revealed by kinase-targeted chemoproteomics. BMC Cancer 2012;12:38.

36 Weigert 0 , Lane AA, Bird L, et al. Genetic resistance to JAK2 enzymatic inhibitors is overcome by HSP90 inhibition. J Exp Med 2012;209:259-73.
37 Reich N, Tomcik M, Zerr P, et al. Jun N-terminal kinase as a potential molecular target for prevention and treatment of dermal fibrosis. Ann Rheum Dis 2012:71:737-45.

38 Yamamoto T, Takagawa S, Katayama I, et al. Animal model of sclerotic skin. I: Local injections of bleomycin induce sclerotic skin mimicking scleroderma. J Invest Dermatol 1999;112:456-62.

39 Green MC, Sweet HO, Bunker LE. Tight-skin, a new mutation of the mouse causing excessive growth of connective tissue and skeleton. Am J Pathol 1976;82:493-512.

40 Saito E, Fujimoto M, Hasegawa M, et al. CD19-dependent B lymphocyte signaling thresholds influence skin fibrosis and autoimmunity in the tight-skin mouse. J Clin Invest 2002;109:1453-62

41 Allegra A, Sant'antonio E, Penna G, et al. Novel therapeutic strategies in multiple myeloma: role of the heat shock protein inhibitors. Eur I Haematol. 2011:86:93-110.

42 Sidera K, Patsavoudi E. HSP9O Inhibitors: current development and potential in cancer therapy. Recent Pat Anticancer Drug Discov 2013. [Epub ahead of print]

43 Kim YS, Alarcon SV, Lee S, et al. Update on Hsp90 inhibitors in clinical trial. Curr Top Med Chem 2009;9:1479-92. 


\section{A RD Heat shock protein 90 (Hsp90) inhibition} targets canonical TGF- $\beta$ signalling to prevent fibrosis

Michal Tomcik, Pawel Zerr, Jana Pitkowski, Katrin Palumbo-Zerr, Jérôme Avouac, Oliver Distler, Radim Becvar, Ladislav Senolt, Georg Schett and Jörg H Distler

Ann Rheum Dis 2014 73: 1215-1222 originally published online May 9, 2013

doi: 10.1136/annrheumdis-2012-203095

Updated information and services can be found at:

http://ard.bmj.com/content/73/6/1215

\section{These include:}

References This article cites 42 articles, 15 of which you can access for free at: http://ard.bmj.com/content/73/6/1215\#BIBL

\section{Email alerting} service

Receive free email alerts when new articles cite this article. Sign up in the box at the top right corner of the online article.

Topic Collections
Articles on similar topics can be found in the following collections

Connective tissue disease (4235)

Immunology (including allergy) (5119)

\section{Notes}

To request permissions go to:

http://group.bmj.com/group/rights-licensing/permissions

To order reprints go to:

http://journals.bmj.com/cgi/reprintform

To subscribe to BMJ go to:

http://group.bmj.com/subscribe/ 